\title{
The Simulation of Bragg-Case Section Images of Dislocations and Inclusions in Aspect of Identification of Defects in SiC Crystals
}

\author{
T. BALCER ${ }^{a}$, W. WierzChOWSKI ${ }^{a}$ AND K. WieteskA ${ }^{b}$ \\ ${ }^{a}$ Institute of Electronic Materials Technology, Wólczyńska 133, 01-919 Warsaw, Poland \\ ${ }^{b}$ Institute of Atomic Energy, 05-400 Otwock-Świerk, Poland
}

\begin{abstract}
The numerical simulation has been applied for studying of Bragg-case section topographic images of dislocation and rod-like inclusions. The validity of simple approximation of extinction contrast was confirmed in the case of screw dislocations in silicon carbide crystals. A procedure for approximate calculation of the strain field of rod-like inclusion was constructed, consisting of adding the contributions from a very large number of point-like inclusions uniformly distributed inside the assumed volume of the inclusion. The procedure ensured a reasonable similarity between the simulated topographs and experimental Bragg-case section topographic images of some pipe-formed cavities in silicon carbide crystals. The method is useful for some other materials, e.g. it enabled to compute realistic simulation of plane-wave topographs of the rod-like inclusions in YAG.
\end{abstract}

PACS numbers: 61.72.Ff, 61.72.up

\section{Introduction}

Bragg-case section topography is an important method of X-ray diffraction topography which can reveal details of strains field of crystallographic defects and provides a possibility of simulation of the topographical images by numerical integration of the Takagi-Taupin equations. The method was initially proposed by Lang $[1,2]$ and was used for observation of the dislocations by ZielińskaRohozińska [3]. Zaumseil [4] observed long-range interference effects in bent crystals and similar interference effects were reproduced in numerical simulations of Bragg-case section images performed by Gronkowski with Bąk-Misiuk et al. [5]. The simulations of dislocation images were described by Wierzchowski et al. [6-8].

The present paper discusses the elements of contrast in the simulated images of dislocation in Bragg-case section topography. In particular, the applicability of the simple approximations of the extinction contrast is examined for screw dislocations perpendicular to the crystal surface in an arbitrary asymmetrical reflection, similarly as in [9]. The simulation of dislocation and rod-like inclusion images were applied for analysis of the Bragg-case section images in silicon carbide crystals, particularly to the images of pipe-formed cavities, suggested by Dudley and coworkers $[10,11]$ as hollow-core super-screw dislocations. Some later papers [12-14] described, however, many cases when the strain fields around micro-pipes cannot be well described by that of hollow-core super-screw dislocation.

An approximate procedure for calculation of the strain field of rod-like inclusion had been revealed, which con- sisted of adding the contribution from a very large number of point-like inclusions uniformly distributed inside the assumed rod volume. The procedure was based on the initial formulae given by Sen [11], and allowed approximate calculation of strains for cylindrical rods perpendicular or inclined to the surface taking into account the relaxation of the strain on free surface.

The necessary completion of the investigations in silicon carbide was testing of the procedure in the case of double-crystal topographs of solute-trails in yttrium aluminum garnet $\left(\mathrm{Y}_{3} \mathrm{Al}_{5} \mathrm{O}_{12}\right)$ [16] exactly corresponding to the assumed model of rod-like inclusions.

\section{Realization of the method and image} simulation in Bragg-case section topography

In the experimental realization of Bragg-case section topographic method the most important point is the use of a beam with a very narrow $5 \mu \mathrm{m}$ wave front. The method can be realized both with the use of conventional and synchrotron sources of X-rays.

The simulation of the topographic images in Bragg-case section topographic method may be performed by numerical integration of the Takagi-Taupin equations. The images may be approximated by spherical wave diffraction pattern with a single point of incidence. More realistic images were presently obtained by adding a number of simulations from the excited neighboring points on the surface, similarly as by Epelboin and Soyer in the case of transmission traverse topographs [17].

The following form of the Takagi-Taupin equations was used [18]: 


$$
\begin{aligned}
& \frac{\partial D_{0}}{\partial s_{0}} \frac{\mathrm{i}}{\pi k}=\chi_{0} D_{0}+C \chi_{\bar{h}} D_{h}, \\
& \frac{\partial D_{h}}{\partial s_{h}} \frac{\mathrm{i}}{\pi k}=C \chi_{h} D_{0}-\alpha_{h} D_{h},
\end{aligned}
$$

where $s_{0}$ and $s_{h}$ are equilateral coordinates, respectively, along the incident and reflected beam directions, $D_{0}, D_{h}$ are generalized wave amplitudes, $\chi_{h} \chi_{0}$ are the coefficients of the Fourier series of the dielectric susceptibility, $C$ is the polarization factor and $\alpha_{h}$ includes the lattice deformation representing the effective change of the Bragg angle

$$
\alpha_{h}=-2 \Delta \Theta \sin 2 \Theta-\chi_{0}-4 \sin \Theta \frac{\partial \boldsymbol{u}(\boldsymbol{r}) \cdot \boldsymbol{h}}{\partial s_{h}},
$$

where $\Delta \Theta$ is the deviation of the incident beam direction from the Bragg angle $\Theta, \boldsymbol{h}$ is the diffraction vector and $\boldsymbol{u}$ is the deformation vector. The last term in (2) represents the effective change of the Bragg caused by the deformation field.

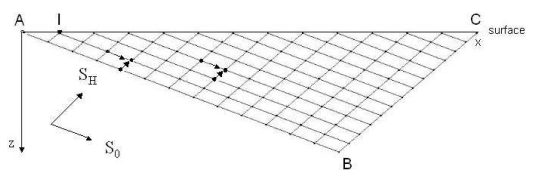

Fig. 1. The integration grid used for integration of the Takagi-Taupin equations in case of Bragg-case section topography with the integration sequence schematically marked by arrows.

The grid used for numerical integration is schematically shown in Fig. 1. The numerical integration was performed using the half-step derivative method proposed by Authier et al. [19] and the way of integration is schematically shown by arrows. Zero values of the generalized amplitudes are taken along the line of grid points $\mathrm{AB}$ on the left side of the excited point I.

\section{Results and discussion}

The simulated Bragg-case section images of the screw-dislocation obtained by numerical integration of the Takagi-Taupin equations are shown in Fig. 2. The first three images were computed for single excited point (ideal spherical wave) and the positions of dislocation chosen in such way that they cut the left side end of the integration grid at $1 / 4,1 / 2$, and $3 / 4$ of its length, which corresponded respectively to the distances $150 \mu \mathrm{m}, 300 \mu \mathrm{m}$ and $450 \mu \mathrm{m}$ from the excited point I. In Figs. 2d-f dislocations are located analogously as in Figs. $2 \mathrm{a}-\mathrm{c}$, but the simulations take into account the finite width of the beam, equal to $5 \mu \mathrm{m}$, by addition of 50 simulations for excited neighboring points of the grid at the surface. A similar method was formerly applied by Epelboin and Soyer [17] for projection topography in the transmission case but to our best knowledge it has not been yet applied in the back-reflection case. The parameters used for simulation correspond to those of $0.06 \mathrm{~nm}$ radiation for $(3 \overline{3} \cdot 16)$ reflections in $\mathrm{SiC}$ with a very low attenuation of $\mathrm{X}$-rays.
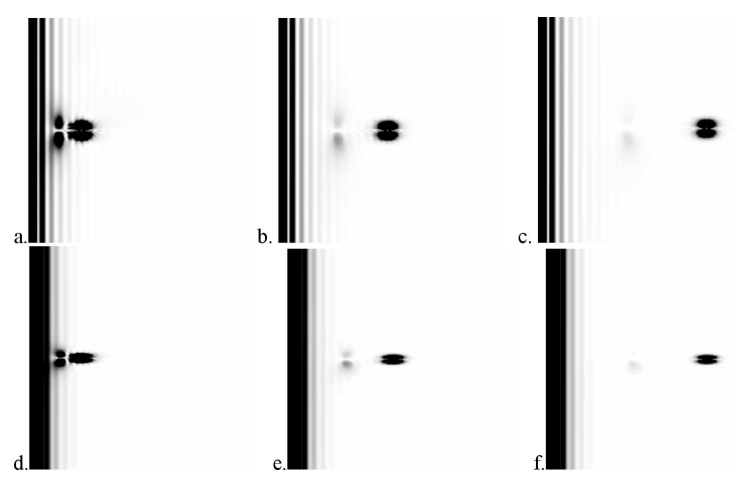

Fig. 2. (a), (b) and (c) Numerical simulations of Bragg-case section images of the screw dislocations perpendicular to the surface and located respectively 150 , 300 and $450 \mu \mathrm{m}$ from the assumed single excited point. (d), (e) and (f) Numerical simulations for the same positions of dislocation as (a), (b) and (c), but taking into account the finite width of the incident beam by adding the contributions from 50 mutually displaced points of incidence. The dimensions of each simulated image are $1600 \mu \mathrm{m} \times 1600 \mu \mathrm{m}$.

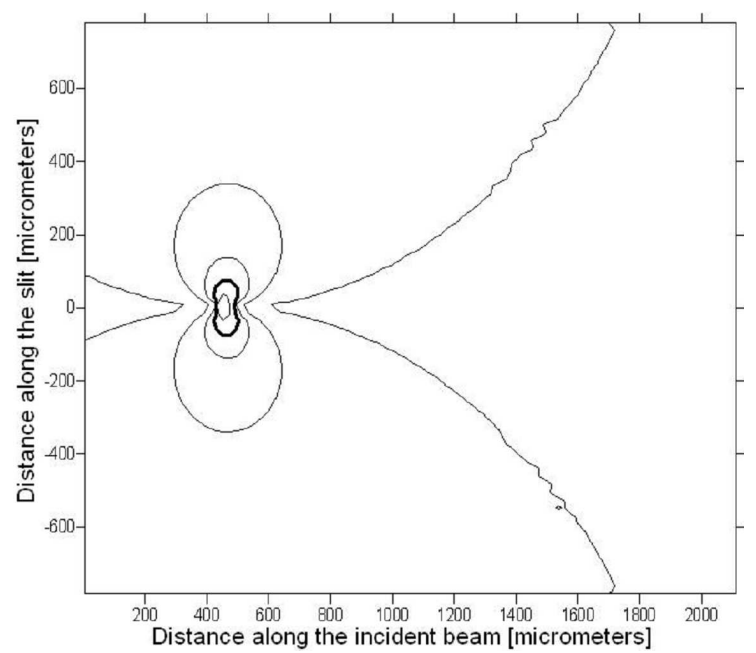

Fig. 3. The distribution of the last term in function $\alpha_{h}$ describing the effective change of the reflecting angle in the plane of the intersecting beam. The thick line corresponds to the condition when the deformation is equal to the width of the rocking curve (3).

As one can notice, the simulated section topographic images of dislocations revealed direct contrast in the form of a black rosette, surrounded by some tails of interference fringes. In simulations taking into account the finite width of the beam the majority of interference effects were dampened and the extension of the images was smaller. Thanks to these changes the character of simulated images better corresponded to the experimental images. 


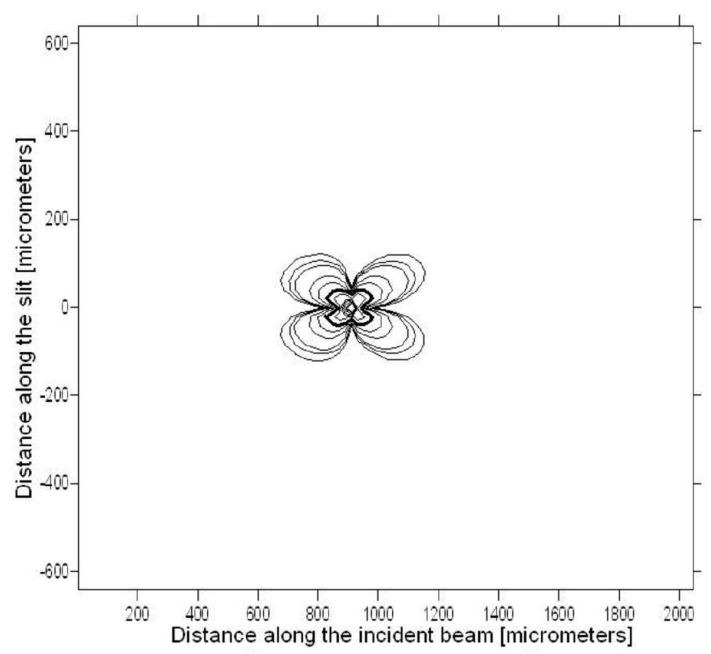

Fig. 4. The distribution of the gradient of $\alpha_{h}$ in the plane of the intersecting beam, characteristic to the prediction of extinction contrast from the condition of the decomposition of wave field. The thick line corresponds to the condition of wave-field decomposition.

It may be noticed that the image of the rosette appears at the point of the surface joined with the point of the intersection of the dislocation with the incident beam along the direction of the reflected beam, as it may be expected in the case of the direct contrast. Only a weak darker contrast is present at the point where the dislocation outcrops the surface, located between a series of the Uragami fringes [20] and the black rosette.

The simulated images of the dislocations were compared with the predictions of two simple models of the extinction contrast described by Miltat and Bowen [9]. The first model assumes that the volume of the deformed lattice, reflecting the radiation as the ideally mosaic crystal and providing additional intensity, is determined by the condition that the change of the reflecting condition shift exceeds the rocking curve width $\delta$, i.e.

$$
4 \sin \Theta \frac{\partial \boldsymbol{u}(\boldsymbol{r}) \cdot \boldsymbol{h}}{\partial s_{h}}>\delta .
$$

The rocking curve width can be calculated from the formula

$$
\delta=\frac{2|C|}{\sin \left(2 \vartheta_{b}\right)} \sqrt{\frac{\gamma_{0}}{\left|\gamma_{h}\right|} \chi_{h} \chi_{\bar{h}}},
$$

where $\gamma_{0, h}$ - direction cosines of the incident and reflected beam wave.

In the case of the second model the volume providing additional intensity is limited by the condition wave field decomposition

$$
\frac{1}{2 \cos \left(\theta_{\mathrm{B}}\right)}\left|\nabla \alpha_{h}\right| \leq \frac{1}{\Lambda^{2}} .
$$

$\Lambda$ is the extinction length

$$
\Lambda=\frac{\lambda \sqrt{\gamma_{0}\left|\gamma_{h}\right|}}{|C| \sqrt{\chi_{h} \chi_{\bar{h}}}}
$$

where $\lambda$ is the radiation wavelength.
The contours coming from these two models calculated in the plane of the intersected beam and corresponding to the simulated image (Fig. 2d) are shown, respectively, in Figs. 3 and 4. As one can notice, the comparison of the simulated images in Fig. 2 with Figs. 3 and 4 confirmed the first model providing the contour of images in form of twofold rosette. Contrary to that, the condition of the wave-field decomposition corresponds to the fourfold rosette.

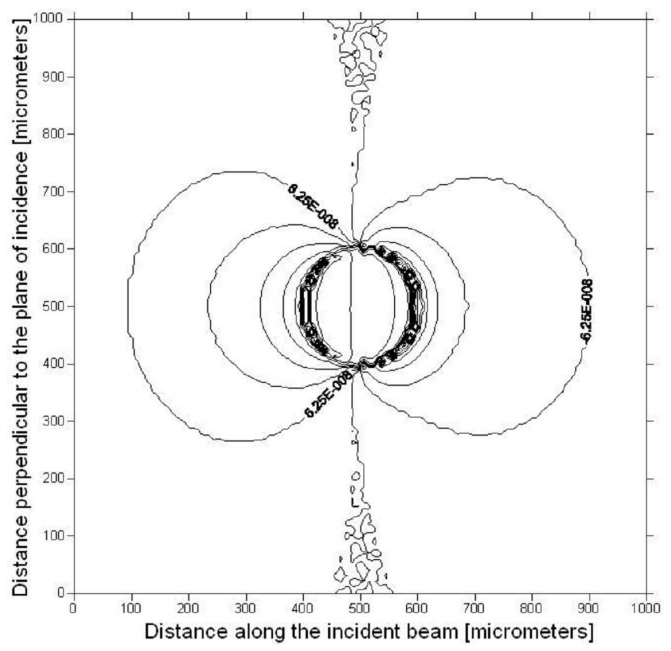

Fig. 5. The computed distribution of the strain fields for the developed model of the rod-like inclusion at the depth of the $10 \mu \mathrm{m}$. The labels of the two levels indicates the change of the sign on two sides of the image.

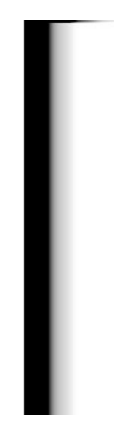

(1)

Fig. 6. The computed Bragg-case section image of the rod-like inclusion. The dimensions of simulated image are $1200 \mu \mathrm{m} \times 1200 \mu \mathrm{m}$.

The calculation of the deformation field of the rod-like inclusion, outcropping the free surface is a complicated problem in the theory of elasticity. The problem was significantly simplified by an approximation based on a simple idea of summation of the contribution from a large number of point-like inclusions regularly distributed inside the volume of inclusion. This idea is justified by the principle of strain superposition, and the use of the formula given by Sen [11] enables taking into account the presence of the free surface. 

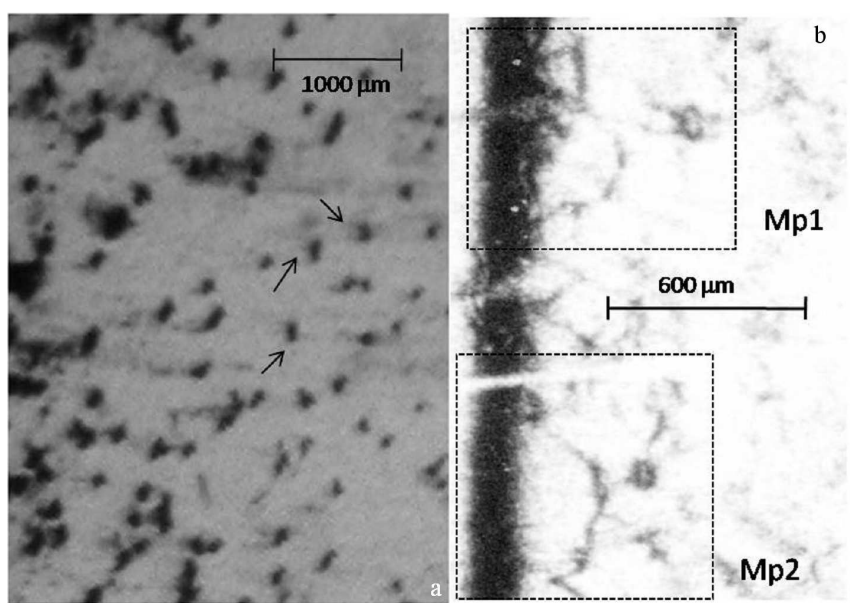

Fig. 7. The experimental synchrotron section images in $\mathrm{SiC}$ crystals (a) with possible images of the screw dislocations marked by arrows, and (b) with intersected pipe-formed cavities M 1 and M 2 (the marked rectangles correspond to the limits of numerical simulation in Fig. 6). Similarly as in all other figures the diffraction plane is located horizontally.

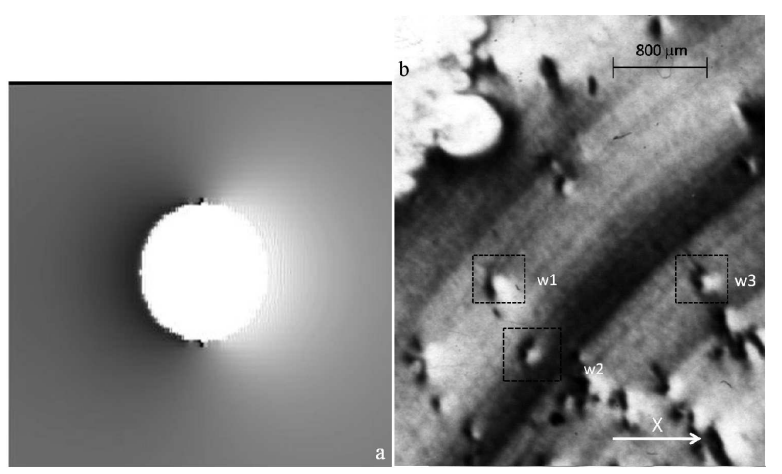

Fig. 8. The comparison of (a) simulated back-reflection plane wave image of the rod-like inclusion and (b) the experimental conventional double-crystal images of the "solute trails" in yttrium aluminum garnet crystals obtained in $620_{\mathrm{Si}}-888_{\mathrm{YAG}}$ setting in $\mathrm{Cu} K_{\alpha_{1}}$ radiation for the high angle flank of the rocking curve.

A significant reduction in the number of the required operations was achieved as follows. (i) At the first stage we calculated the contribution from inclusions located along the line, being the assumed direction of the rod-like inclusions, and stored the values in a very large $400 \times$ $400 \times 200$ matrix. (ii) In the next stage it was possible to calculate the resulting field at a particular point by adding the neighboring values at the same depth in a plane defined by third index from the area defined by the assumed section of the rod-like inclusions.

The representative contours of the effective change of reflecting condition computed with the revealed procedure are shown in Fig. 5. They retain the axial character of the obtained field with the change of the sign along the reflecting planes and they provide almost constant value of the strain inside the inclusion. The representative simulation of the Bragg case section for the rod-like inclusion perpendicular to the surface is shown in Fig. 6. The obtained contrast was of extinction type in the form of two asymmetrically broken parts of the ring, coming from the region where the incident beam intersects the inclusion.

The representative experimental synchrotron section images of $4 \mathrm{H}$ silicon carbide bulk crystals with a relatively low concentration of defects are shown in Fig. 7. It may be expected that most of the observed dislocations are of screw type. As may be noticed in the section topographs shown in Fig. 7a, many dislocations provide the extinction contrast in the form of the twofold rosette, but often not quite symmetrical along the diffraction plane.

The contrast at the two micro-pipes, present in Fig. 7b, and marked with the dotted rectangle is very similar to the simulated image shown in Fig. 6. It should be noted that the micro-pipe is not exactly corresponding to the presently assumed rod-like inclusions, but one can expect a significant similarity of the strain field around the rod-like inclusion and pipe-formed cavity with a significant tensile or comprehensively strained shell on the inner surface. On the other hand, one can rather exclude the possibility that the pipes in Fig. 7b are hollow-core super-screw dislocations, in view of a considerably different character of the formerly discussed images of screw dislocation.

The additional test of the revealed procedure was performed in the case more strictly corresponding to the assumed model of the inclusions, namely that of double crystal topographic images of the solute trails in yttrium aluminum garnet crystals, similar to those discussed in [16] obtained in $620_{\mathrm{Si}}-888_{\mathrm{YAG}}$ setup in $\mathrm{Cu} K_{\alpha_{1}}$ radiation.

The simulated multi-crystal (plane-wave) image shown in Fig. 8a is to a great extent reproducing the character of the strain field distribution shown in Fig. 5. One can notice that the central region corresponds to the angular shift far from the diffraction peak and is practically not reproduced, similarly as in the case of the Bragg-case section images of inclusion in Fig. 6.

\section{Conclusions}

Taking into account the finite width of the beam provided more realistic simulations of the dislocation images. A possibility of simple approximation of extinction contrast from the condition of exceeding the reflection range was confirmed.

A procedure for approximate calculation of the strain field of rod-like inclusions was constructed. The procedure allowed taking into account the relaxation of strain on the free surface, for cylindrical rods perpendicular or inclined to the free surface. It ensured a realistic simulation of section and plane-wave topographs of the rod-like inclusions, and obtaining of reasonable similarity to the experimental Bragg-case section topographic images of some pipe formed cavities in $\mathrm{SiC}$. 


\section{References}

[1] A.R. Lang, Acta Crystallogr. 7, 583 (1954).

[2] A.R. Lang, Z.-H. Mai, Proc. R. Soc. A 368, 313 (1979).

[3] E. Zielińska-Rohozińska, Phys. Status Solidi A 59, 317 (1980).

[4] P. Zaumseil, Kristall u. Technik 13, 963 (1978).

[5] J Bąk-Misiuk, J. Gronkowski, J. Härtwig, W. Wierzchowski, Phys. Status Solidi A 99, 345 (1987).

[6] W. Wierzchowski, M. Moore, Acta Phys. Pol. A 82, 193 (1992).

[7] W. Wierzchowski, K. Wieteska, W. Graeff, Il Nuovo Cimento 19D, 233 (1997).

[8] W. Wierzchowski, K. Wieteska, W. Graeff, J. Phys. D, Appl. Phys. 33, 1230 (2000).

[9] J.E. Miltat, D.K. Bowen, J. Appl. Crystallogr. 8, 657 (1975).

[10] M. Dudley, X.R. Huang, V.M. Vetter, J. Phys. D, Appl. Phys. 36, A30 (2003).
[11] X.R. Huang, M. Dudley, W.M. Vetter, W. Huang, S. Vang, C.H. Carter, Appl. Phys. Lett. 74, 353 (1999).

[12] H. Obsato, T. Kato, T. Okuda, Mater. Sci. Semicond. Process 4, 483 (2001).

[13] T. Argunova, V. Kohn, J.-W. Jung, J.-H. Je, Phys. Status Solidi A 206, 1833 (2009).

[14] V. Kohn, T. Argunova, J.-H. Je, Appl. Phys. Lett. 91, 171901 (2007).

[15] R. Sen, J. Quant. Mech. VIII, 365 (1949).

[16] K. Mazur, W. Wierzchowski, J. Cryst. Growth 148, 345 (1995).

[17] Y. Epelboin, A. Soyer, Acta Crystallogr. A 41, 61 (1985).

[18] T. Bedyńska, Phys. Status Solidi A 18, 147 (1973).

[19] A. Authier, C. Malgrange, M. Tournarie, Acta Crystallogr. A 24, 126 (1968).

[20] T. Uragami, J. Phys. Soc. Japan 27, 147 (1969). 